\title{
Long-term restoration success of insect herbivore communities in seminatural grasslands: a functional approach
}

\author{
Felix Neff (D) $1,2,4$ M. Carol Resch (iD) ${ }^{3}$ Anja Marty, ${ }^{3}$ Jacob D. Rolley, ${ }^{3}$ Martin Schütz, ${ }^{3}$ Anita C. Risch (D), ${ }^{3}$ \\ AND MARTIN M. GosSNER (D) 1 \\ ${ }^{1}$ Forest Entomology, Swiss Federal Research Institute WSL, Birmensdorf 8903 Switzerland \\ ${ }^{2}$ Landscape Ecology, Institute of Terrestrial Ecosystems, ETH Zürich, Zürich 8092 Switzerland \\ ${ }^{3}$ Community Ecology, Swiss Federal Research Institute WSL, Birmensdorf 8903 Switzerland
}

Citation: Neff, F., M. C. Resch, A. Marty, J. D. Rolley, M. Schütz, A. C. Risch, and M. M. Gossner. 2020. Long-term restoration success of insect herbivore communities in seminatural grasslands: a functional approach. Ecological Applications 30(6):e02133. 10.1002/eap.2133

Abstract. Seminatural grasslands are important biodiversity hotspots, but they are increasingly degraded by intensive agriculture. Grassland restoration is considered to be promising in halting the ongoing loss of biodiversity, but this evaluation is mostly based on plant communities. Insect herbivores contribute substantially to grassland biodiversity and to the provisioning of a variety of ecosystem functions. However, it is unclear how they respond to different measures that are commonly used to restore seminatural grasslands from intensively used agricultural land. We studied the long-term success of different restoration techniques, which were originally targeted at reestablishing seminatural grassland plant communities, for herbivorous insect communities on taxonomic as well as functional level. Therefore, we sampled insect communities $22 \mathrm{yr}$ after the establishment of restoration measures. These measures ranged from harvest and removal of biomass to removal of the topsoil layer and subsequent seeding of plant propagules. We found that insect communities in restored grasslands had higher taxonomic and functional diversity compared to intensively managed agricultural grasslands and were more similar in composition to target grasslands. Restoration measures including topsoil removal proved to be more effective, in particular in restoring species characterized by functional traits susceptible to intensive agriculture (e.g., large-bodied species). Our study shows that long-term success in the restoration of herbivorous insect communities of seminatural grasslands can be achieved by different restoration measures and that more invasive approaches that involve the removal of the topsoil layer are more effective. We attribute these restoration successes to accompanying changes in the plant community, resulting in bottom-up control of the herbivore community. Our results are of critical importance for management decisions aiming to restore multi-trophic communities, their functional composition and consequently the proliferation of ecosystem functions.

Key words: attribute diversity; functional traits; herbivorous insects; long-term recovery; restoration ecology; seminatural grasslands; topsoil removal.

\section{INTRODUCTION}

Biodiversity is decreasing at an alarming rate worldwide with land-use intensification and associated habitat loss being the major drivers of this decline (Sala et al. 2000, Newbold et al. 2015). In Central Europe, seminatural grasslands are important biodiversity hotspots, but their unique and diverse plant and animal communities are increasingly under threat (Török and Dengler 2018). Mostly, intensified agricultural practices such as the use of synthetic fertilizer, frequent plowing with subsequent seeding of standardized plant species-poor seed mixtures, and intensive mowing or grazing (Temme and

Manuscript received 30 October 2019; revised 11 February 2020; accepted 17 March 2020. Corresponding Editor: Matthew P. Ayres.

${ }^{4}$ E-mail: felix.neff@wsl.ch
Verburg 2011) hamper biodiversity and lead to biotic homogenization across multi-trophic groups of organisms at different spatial scales (Manning et al. 2015, Gossner et al. 2016). The decline in insect biomass and diversity is an example of apparent loss of higher-trophic biodiversity that is currently intensively discussed (Habel et al. 2016, Hallmann et al. 2017, Sánchez-Bayo and Wyckhuys 2019, Seibold et al. 2019). Halting this ongoing loss has become a political and societal mandate (IPBES 2019). To achieve this, strategies and programs, such as agri-environmental schemes, have been developed. Because most seminatural grasslands in Central Europe were lost in the recent decades (Gimmi et al. 2011, Gattlen et al. 2017), additional restoration projects using different methods, such as reintroduction of grazing ("rewilding"), harvest and removal of biomass, or removal of the topsoil layer, were implemented on 
various occasions (Marrs et al. 1998, Kiehl and Pfadenhauer 2007, Frouz et al. 2009, Resch et al. 2019). Topsoil removal is a reliable tool to reduce both high nutrient amounts in soils and the concomitant competitive advantage of non-target plant species over target species (Verhagen et al. 2001, Allison and Ausden 2004, Power et al. 2006).

Insects contribute substantially to the diversity of seminatural grasslands, are important drivers of energy and nutrient cycling and serve as major food source for tertiary consumers such as bats, birds, and small mammals. A recent study on multifunctionality in Central European grasslands showed, for example, that insect herbivores were the most important drivers of ecosystem functioning among nine studied plant and animal trophic groups (Soliveres et al. 2016). A variety of studies investigated the success of restoration measures for insect communities. For example, many studies have assessed how low-impact grassland management (no fertilization, removal of biomass; e.g., Nickel and Achtziger 2005, Karg et al. 2015), reintroduction of grazing (WallisDeVries and Raemakers 2001, Öckinger et al. 2006), or sowing of plant propagules from target communities in agricultural land (Woodcock et al. 2010, 2012, Rowe and Holland 2013) affect the success of insect community restoration. In addition, it has been stressed that the landscape context is crucial for the outcome of restoration measures (Woodcock et al. 2010, Zirbel et al. 2019). However, these assessments (1) did not consider more invasive restoration measures, such as topsoil removal, that may impact the long-term recovery of insect communities and (2) normally focused only on the taxonomic dimension of insect communities and did not consider functional approaches (but see Verhagen et al. 2008). It therefore remains unclear whether the insect community's composition and functional breadth can be reestablished with different restoration regimes.

The functional role of species in an ecosystem is mediated by their functional traits. A functional perspective using trait-based approaches is thus a step forward in understanding human impacts on insect community assembly and allows to obtain more general results (McGill et al. 2006). Consequently, such an approach is expedient to explore whether the functional composition of communities can be restored. In general, species need to overcome several filters before establishing on a restored grassland site (Keddy 1992). Specifically, dispersal filters determine which species reach a site whereas biotic and abiotic filters determine which species establish given a particular environmental setting and the presence of other organisms (Belyea and Lancaster 1999). In grasslands, intensified agriculture at local and landscape scale has been shown to act as a filter selecting against large-bodied species and habitat specialists with restricted dispersal capacities during community assembly (Ribera et al. 2001, Rader et al. 2014, Gámez-Virués et al. 2015, Simons et al. 2016, Neff et al. 2019). Consequently, trait combinations that suffer most from intensified land use might be particularly difficult to restore (Woodcock et al. 2012). Moreover, depending on the species that arrive first, different trajectories of the development of species assembly may result (i.e., priority effects; e.g., Young et al. 2005), leaving it unclear whether restoration measures will lead to a similar functional state of the communities after a particular time.

In this study, we evaluated the long-term success of different restoration measures in reestablishing taxonomic and functional diversity and composition of insect herbivore communities. We used the unique opportunity of a large-scale restoration project established $22 \mathrm{yr}$ ago, in which the success of three different restoration measures (harvest of biomass only, topsoil removal, topsoil removal with addition of plant propagules) in restoring seminatural grassland plant communities from intensively managed agricultural land was evaluated (Resch et al. 2019). As in other study systems (Kiehl et al. 2010), measures involving topsoil removal proved to be most effective in restoring species-rich plant communities (Resch et al. 2019), as they successfully reduce excessive soil nutrients. Here, we assessed herbivorous insect communities in intensively managed grasslands (i.e., initial state), in grasslands under the three aforementioned restoration measures, as well as in remnants of seminatural grasslands (i.e., target state). We hypothesized that, $22 \mathrm{yr}$ after restoration, (1) taxonomic and functional diversity as well as composition of herbivorous insect communities of all restoration measures would be developed toward the target state; (2) more invasive restoration measures would be more successful in restoring the target state insect communities (Harvest only $<$ Topsoil $<$ Topsoil + Propagules), following trends found for plant communities; and (3) insect species with trait characteristics and trait combinations that respond negatively to land-use intensification would be successfully restored, but success would depend on the applied restoration measure (Harvest only $<$ Topsoil < Topsoil + Propagules; see Appendix S1: Tables S2, S3 for detailed hypotheses).

\section{Materials And Methods}

\section{Study site}

The study area was situated within and nearby to Eigental nature reserve $\left(47^{\circ} 27^{\prime} 36^{\prime \prime}\right.$ to $47^{\circ} 29^{\prime} 06^{\prime \prime} \mathrm{N}$, $8^{\circ} 37^{\prime} 12^{\prime \prime}$ to $8^{\circ} 37^{\prime} 44^{\prime \prime}$ E, $461-507 \mathrm{~m}$ above sea level) in the vicinity of Zurich airport (Canton Zurich, Switzerland). Mean annual precipitation and temperature is $903 \pm 136 \mathrm{~mm}$ and $9.14^{\circ} \pm 0.50^{\circ} \mathrm{C}$ (mean $\pm \mathrm{SD}$ for 2007-2017; MeteoSchweiz 2018). In 1967, the Eigental nature reserve was established to protect small and isolated remnants of species-rich, seminatural grasslands (roughly $12 \mathrm{ha}$ ), which were embedded in an otherwise intensively managed landscape. It is characterized by oligo- to mesotrophic Molinion (semi-wet, matrix 
species Molinia caerulea) and Mesobromion (semi-dry, matrix species Bromus erectus) meadows (Delarze et al. 2015), reflecting small-scale habitat heterogeneity, mainly due to site-specific groundwater levels and slope inclination. As in most Central European grasslands, management is necessary to prevent shrub and tree invasions as well as to secure low levels of available soil nutrients and thus to maintain these species-rich habitats (Poschlod and WallisDeVries 2002).

In 1990, the government of the Canton Zurich decided to enlarge the Eigental nature reserve as a counter measure against degradation and biodiversity loss in seminatural grasslands due to overutilization and the excessive input of nutrients (mostly nitrogen). Eleven patches of adjacent intensively managed grassland (in total roughly $20 \mathrm{ha}$ ) were targeted to be transformed into seminatural grasslands (see Appendix S1: Fig. S1 for a detailed map). As a first restoration measure, fertilization was ceased, and biomass harvested three times to remove excessive soil nutrients from the original system and thus benefit plant species with low competitive ability on the long run. In 1995, the restoration efforts were increased and a large-scale experiment comprising three restoration measures with increasing intervention intensities was implemented (see Appendix S1: Table S1 for details): (1) Harvest only. Initial restoration measures were continued with mowing and removing of the aboveground biomass two times a year (early summer and autumn). (2) Topsoil. Removal of topsoil, depending on the thickness of the A horizon the upper 10-20 cm, in four randomly selected areas within the 11 patches in late autumn 1995. The size of the restoration area depended on individual patch size $\left(2,700-7,000 \mathrm{~m}^{2}\right)$. (3) Topsoil + Propagules. Plant propagules were added on one-half of the area where topsoil was removed via application of fresh, seed-containing hay and hand-collected propagules of target species originating from semi-dry and semi-wet species-rich grasslands with local and regional provenance (within radius of 7-30 km) (1995, 1996, 1997; for further details see timeline in Appendix S1: Fig. S2, but also Resch et al. 2019).

Management of Topsoil and Topsoil + Propagules started $5 \mathrm{yr}$ after treatment implementation and included yearly mowing and removing of aboveground biomass (late summer or early autumn, also see Appendix S1: Table S1). The experiment was complemented with intensively managed grassland sites that share the same agricultural history as the restored sites (Initial; swards dominated by Lolium perenne, L. multiflorum, and Trifolium repens): mowing and subsequent fertilizing (manure) up to five times a year, as well as different tillage regimes (Appendix S1: Table S1). Finally, sites were selected in target semi-dry and semi-wet grasslands (Target) located within the Eigental nature reserve and another nature reserve nearby (Altläufe der Glatt; $47^{\circ} 27^{\prime} 41^{\prime \prime}$ to $47^{\circ} 28^{\prime} 29^{\prime \prime} \mathrm{N}, 8^{\circ} 31^{\prime} 56^{\prime \prime}$ to $8^{\circ} 32^{\prime} 26^{\prime \prime} \mathrm{E}, 418$ $420 \mathrm{~m}$ above sea level). The selected Target sites are mown and aboveground biomass removed once a year in late summer or early autumn. For each of the five treatments, we selected 11 plots $(5 \times 5 \mathrm{~m})$ spread across the sites. Altogether, the experiment included 55 plots (Appendix S1: Table S1, Fig. S1).

\section{Arthropod sampling}

Aboveground arthropods were sampled using suction sampling on four consecutive days in early July 2017 before the grasslands were mown. This sampling time is ideal to cover a large proportion of our focal species in the adult stage (see Appendix S1: Fig. S3). Arthropods were sampled in two locations on each $5 \times 5 \mathrm{~m}$ plot, once in the southwestern and once in the northeastern corner to account for possible spatial heterogeneity within the plots (details in Appendix S1). Because of the small plot sizes and thus the proximity of sampling plots in some cases, we refrained from repetitive sampling. Repeated sampling would have probably been impacted by previous sampling efforts, i.e., by the removal of the juvenile stages. By removing the settled species, the second sampling would likely have been dominated by more mobile re-immigrants from the surrounding. We additionally tested for low coverage of species with certain phenologies and analyzed sample completeness (Chao and Jost 2012), and found no indication for biased sampling and high sample coverage values for all treatments (Appendix S1: Figs. S3-S5). Thus, we are confident that the data presented is highly robust. The two subsamples per plot were pooled and arthropods were sorted to order or lower taxonomic levels. Here, we focused on three herbivorous taxa (Hemiptera: Auchenorrhyncha; Hemiptera: Heteroptera; Orthoptera), which constitute a large part of the insect herbivore community in seminatural grasslands (e.g., Neff et al. 2019). Individuals were identified to species level (details in Appendix S1).

\section{Functional traits}

We used two sets of functional traits in this study (Appendix S1: Tables S2, S3). First, we used eight morphometric traits: body volume, body shape, hind femur shape, hind/front leg ratio, wing length, leg length, antenna length, and eye width. These traits are linked to four different functions, which potentially determine species' responses to land use and habitat restoration: dispersal, disturbance tolerance/avoidance, microhabitat use, and resource consumption. Second, we used six lifehistory traits, which were based on an existing data set collected by Gossner et al. (2015). We included traits describing different life-history characteristics of herbivore insect species, namely: feeding specialization, feeding tissue, hibernation stage, and number of generations per year, which are related to insect species' vulnerability to changes in plant community composition, microhabitat use, and disturbance tolerance. To represent potential changes in habitat moisture with abandonment of intensive land use (e.g., change in ground-water level), we also 
included two traits related to preferred habitat moisture of the study species: moisture preference, describing species' optimum habitat moisture, and moisture range, which describes the species' range of preferable moisture conditions. Depending on the trait values, life-history traits were encoded nominally, ordinally or numerically. See Appendix S1 (Tables S2, S3) for details on functional trait measurements.

\section{Statistical analyses}

To address our hypotheses 1 and 2, we analyzed differences in the (functional) diversity and composition of the insect herbivore communities between treatments based on attribute diversity and principal coordinate analyses. To address hypothesis 3 , we further investigated which functional traits were changing between communities by using RLQ analyses. To exclude the possibility that the observed patterns are based solely on differences in the relative abundance of the three insect groups, the analyses were performed for the combined data set and for a subset only including auchenorrhynchan species. Separate analyses for the other two groups (Heteroptera, Orthoptera) were not possible because of too low individual numbers per sample. The results for Auchenorrhyncha (see Appendix S1: Tables S10, S11, Figs. S17-S23) and for the whole data set were similar and we show the results for all three groups combined in the main text. Due to the clustered study design of the experiment (Appendix S1: Fig. S1) and to account for potential confounding factors, we run a set of linear mixed-effect models and linear models for diversity and composition metrics (Appendix S1 for details). These helped us to be confident that our results were not affected by confounding factors, the nested structure and spatial arrangement of the study plots (Appendix S1: Tables S5, S6, Figs. S6-S8). For this reason, we did not further account for them in the analyses presented within the main text. All analyses were performed in R 3.5.2 (R Core Team 2018).

\section{Attribute diversity}

To test for differences in both taxonomic and functional diversity of communities between the treatments, we applied a newly developed method that allows to construct functional diversity profiles from species abundances based on so-called attribute diversity. This is a generalization of Hill numbers of order $q$ (Chao et al. 2014), where $q$ represents the strength by which common entities are considered and allows to quantify diversity with different weighting of the functional distances between species (Chao et al. 2019). With this approach, we were able to quantify differences in diversity between treatments for a gradient of weighing of functional trait information and a gradient of weighing of common species. The method is based on species' pairwise functional dissimilarities, which are truncated at different levels of a threshold distinctiveness value $\tau$ to weigh large functional dissimilarities differently: at the minimum dissimilarity value $d_{\min }$, all species are considered equally different (i.e., attribute diversity equals taxonomic species diversity); at the mean dissimilarity value $d_{\text {mean }}$, functional distances above the mean dissimilarity value are not weighted more strongly in calculation of functional diversity; and at the maximum dissimilarity value $d_{\max }$, none of the dissimilarity values is truncated, corresponding to classically used functional diversity metrics (e.g., Rao's $Q$ ). Here, our main focus was on overall differences between treatments. Thus, we determined attribute $\gamma$-diversity for each treatment from summed species abundances for different values of $q(0 \leq q \leq 3)$ and different values of $\tau\left(d_{\min }, d_{\text {mean }}, d_{\max }\right)$ based on morphometric and life-history traits. Additionally, to test whether changes in $\gamma$-diversity are reflected by changes at the plot level, we determined attribute $\alpha$-diversity (mean of attribute diversity for single communities) and attribute $\beta$-diversity (attribute $\gamma$-diversity divided by attribute $\alpha$-diversity) for all treatments and trait data sets. All analyses of attribute diversity were based on R code provided by Chao et al. (2019).

We hypothesized that changes in insect herbivore communities follow changes in the plant communities. To test this, we analyzed pathways linking the restoration measures and attribute diversity by using piecewise structural equation modelling (Lefcheck 2016) with a set of parameters describing the environmental condition as well as the vegetation of the plots. Details and results are provided in Appendix S1 (Table S7, Figs. S11, S12).

\section{Community composition}

Because the (functional) diversity of the insect communities could be restored without restoring the insect composition of the target community (i.e., promoting a very different but still diverse community), we tested for differences in taxonomic and functional community composition between treatments. We performed principal coordinate analyses $(\mathrm{PCoA})$ on pairwise community dissimilarities between plots. Taxonomic dissimilarity was based on Bray-Curtis distances calculated from species abundances. To calculate functional community dissimilarities, we used the trait probability density (TPD) framework introduced by Carmona et al. (2016), which allows us to determine functional dissimilarity metrics based on the overlay of probabilistic multidimensional hypervolumes. Because the framework was developed for a maximum of four trait dimensions, we reduced both the morphometric and the life-history traits to four trait dimensions using ordination techniques in the ade 4 package (Dray et al. 2018). For morphometric traits, we used principal component analyses whereas, for life-history traits, we used ordination of mixed quantitative variables and factors. Because the TPD framework allows to incorporate intraspecific trait variability, which is lacking from our data sets, we scaled each ordination 
trait axis to standard deviation of 1 and species-level hypervolumes were then estimated from the scaled trait values and a fixed standard deviation of 0.5 (Lamanna et al. 2014). From the resulting hypervolumes, pairwise community dissimilarities were calculated. All TPD analyses were performed using the package TPD (Carmona 2017).

Based on the community dissimilarities, we performed PCoA using the package ape (Paradis et al. 2019) to visualize differences in community composition between treatments. To test for significant treatment effects, we additionally performed permutational multivariate analysis of variance (PerMANOVA) using the package vegan (Oksanen et al. 2018) including all five treatments and for all pairwise comparisons between treatments. As for attribute diversity, we tested pathways, through which the restoration measures were affecting the community composition, using piecewise structural equation modelling (Lefcheck 2016). Details and results are provided in Appendix S1 (Table S7, Figs. S11, S13).

\section{Trait syndromes}

To test which traits and trait combinations were affected by the treatments, we used RLQ analysis (Dolédec et al. 1996). This method allows to identify trait syndromes of species sharing similar traits and reacting similarly to different treatments. It has the advantage over testing each trait separately in that it accounts for different traits being linked, e.g., through trade-offs or phylogenetic linkage. We included treatment as environmental variable ( $\mathrm{R}$ table), the species' morphometric traits or life-history traits as trait variables (Q table), and Hellinger transformed species abundances ( $\mathrm{L}$ table). Ordinal life-history traits were encoded as numeric variables, because RLQ analysis is not possible for trait data sets including ordinal variables. Based on the three tables, we performed RLQ analysis using the package ade4 (Dray et al. 2018). From Euclidean distances based on ordination axes 1 and 2, we identified species clusters using Ward's hierarchical clustering. The optimal number of clusters was determined with the Caliński-Harabasz stopping criterion (Caliński and Harabasz 1974). We restricted the optimal number of clusters to values between two and four for better interpretability of results. Clustering analyses were implemented following Kleyer et al. (2012). Additional to RLQ analysis, we determined community weighted means for all traits and plots with the package FD (Laliberté et al. 2014).

\section{RESULTS}

The analyses were based on 1,063 individuals (mean \pm SD: $19.3 \pm 18.8$ individuals per plot) of 86 species $(7.2 \pm 3.5$ species per plot), of which 911 (16.6 \pm 18.6 individuals per plot) were auchenorrhynchans belonging to 57 species $(5.3 \pm 3.1$ species per plot), $86 \quad(2.2 \pm 1.7$ individuals per plot $)$ were heteropterans belonging to 22 species $(1.4 \pm 0.8$ species per plot) and $66(2.0 \pm 0.9$ individuals per plot $)$ were orthopterans belonging to 7 species $(1.4 \pm 0.6$ species per plot; see Appendix S1: Table S4 for complete species list).

\section{Attribute diversity}

We found significant increases in attribute $\gamma$-diversity for all three restoration measures (Harvest only, Topsoil, Topsoil + Propagules) compared to Initial (Fig. 1), but diversity levels were similar between our three restoration measures and Target. This effect was evident for all levels of the distinctiveness threshold $\tau$, indicating that both taxonomic as well as functional diversity of both morphometric and life-history traits have been restored. The shapes of the diversity profiles along the gradient of order $q$ were similar. However, the decrease in attribute diversity with increasing order $q$ tended to be less steep for low values of $\tau$ in Target, indicating higher taxonomic evenness in Target compared to the three restoration treatments. Furthermore, there was a tendency toward higher attribute diversity in Topsoil + Propagules compared to Harvest only and Topsoil.

Decomposition of attribute in its $\alpha$-, $\beta$ - and $\gamma$-components showed that the effects found for attribute $\gamma$-diversity were reflected by changes in $\alpha$-diversity (Appendix S1: Figs. S14, S15). The exception was Harvest only, for which $\alpha$-diversity was similarly low as for Initial, particularly for functional diversity based on morphometric traits. For low $q$-values, Target also showed similarly low levels of $\alpha$-diversity, which was leveled out at higher $q$-levels as indicated by a less steep decrease of the Target diversity profile. These discrepancies between $\alpha$ - and $\gamma$-diversity coincide with high levels of $\beta$-diversity in Harvest only and Target (at low $q$ ).

The structural equation models revealed both direct and indirect pathways that link restoration treatments and attribute diversity (Appendix S1: Fig. S12). Indirect pathways between restoration treatments and attribute diversity involved vegetation height, plant community composition, biomass of forbs and surface temperature. Generally, direct relationships became stronger with increasing values of $\tau$.

\section{Community composition}

We found a significant treatment effect on community composition for both taxonomic and functional metrics (Fig. 2, Table 1). This was mainly due to a significantly different community composition in Initial compared to all other treatments (Appendix S1: Table S8). Additionally, Harvest only tended to be between Initial and the other treatments in terms of both taxonomic and functional community composition, and this observation was particularly pronounced for taxonomic community composition. However, none of the three restoration 

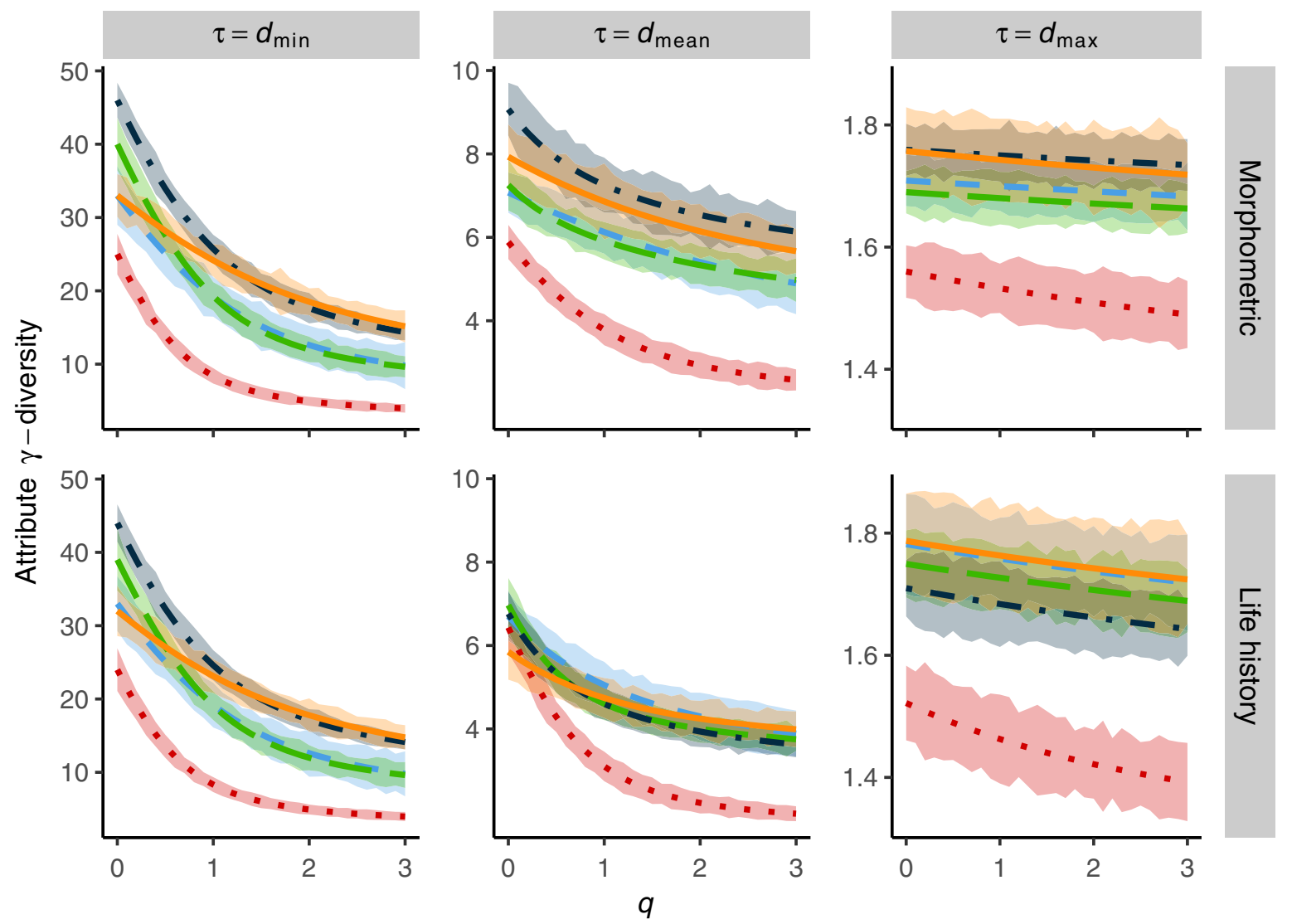

\section{... Initial - - Harvest only - - Topsoil - - . Topsoil + Propagules $=$ Target}

FIG. 1. Diversity profiles of attribute $\gamma$-diversity for different threshold distinctiveness levels $(\tau)$ and different trait data sets (morphometric, life history) for each of the five treatments (indicated by different line colors). Each plot shows the attribute diversity along increasing levels of order $q$, reflecting increasing weighting of common species $(q=0$ corresponds to richness). The threshold distinctiveness level $\tau$ varies between $d_{\min }$ (attribute diversity corresponds to taxonomic diversity, except species pairs with exactly the same traits, which are counted as one species), $d_{\text {mean }}$ (dissimilarity values greater than the mean pairwise dissimilarity value $d_{\text {mean }}$ are truncated), and $d_{\max }$ (pairwise functional dissimilarities are not truncated and thus considered along the whole range of functional dissimilarity values). The uncertainty is calculated based on 50 bootstraps.

measures was found to be significantly different from Target based on pairwise comparisons (Appendix S1: Table S8).

Structural equation models revealed that the effects of restoration treatments on insect community composition were mediated through different aspects of the plant community, i.e., mainly plant community composition, but also diversity and biomass of forbs (Appendix S1: Fig. S13).

\section{Trait syndromes}

The RLQ analysis on morphometric traits revealed four clusters of functionally similar species reacting to the restoration measures in concert (Fig. 3). Cluster 1 was particularly associated with Initial and was characterized by long-winged and long-legged species. Cluster 2 was preferably found in Target as well as Topsoil + Propagules and contained thin-bodied insects with short appendages but relatively long hind legs. Cluster 3 was strongly related to Harvest only and was characterized by small insect with long antennae and wide eyes. In contrast, cluster 4 was strongly associated with Topsoil and contained very large species with small eyes. Generally, ordination axis 1 , which separated Initial and Harvest only (low values) from the other treatments (high values), was most strongly associated with an increase in body volume, as well as a decrease in wing-, leg-, and antenna length.

For life-history traits, RLQ analysis resulted in three clusters (Fig. 4). Cluster 1 showed a strong association with Initial and was characterized by phloem-sucking feeding specialists with more than one generation a year. Cluster 2, which was preferably found in Target, Topsoil and Topsoil + Propagules, contained insects with a variety of different feeding habits that mostly overwinter in the egg stage, only develop one generation per year and have preference for moist conditions. In contrast, cluster 


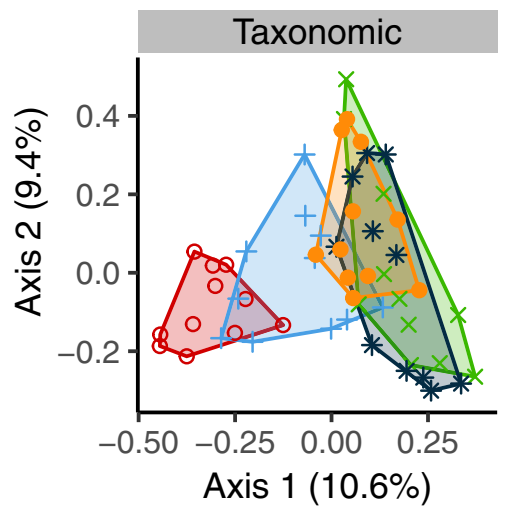

0 Initial + Harvest only
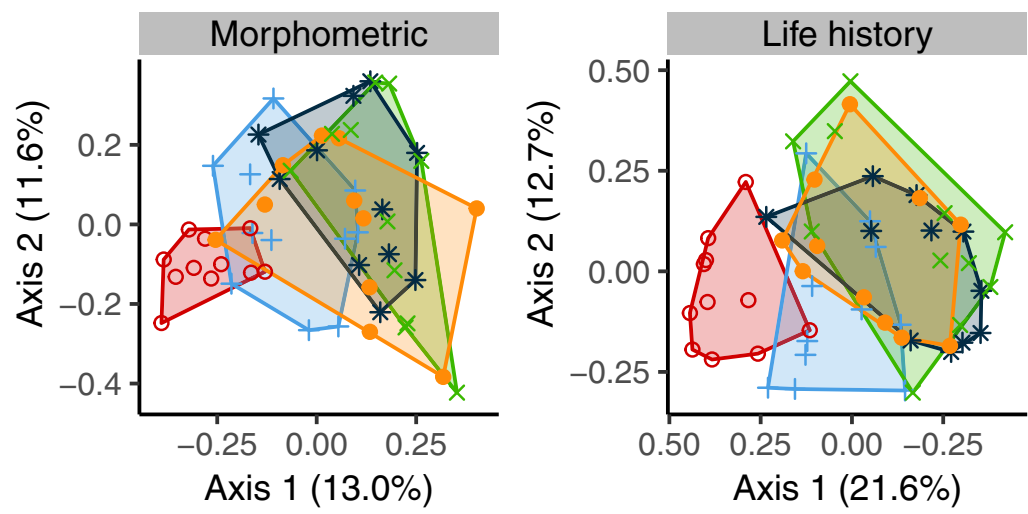

FIG. 2. Biplots of the first two axes from principal coordinate analyses for different sets of pairwise dissimilarities between herbivorous insect communities. Each point represents a community; colors and shapes denote the five treatments. The enclosing shapes are convex hulls. Taxonomic dissimilarities are Bray-Curtis distances calculated from species abundances, whereas functional dissimilarities for morphometric and life-history traits were calculated based on the trait probability density framework.

TABle 1. Permutational multivariate analysis of variance on dissimilarities in community composition, analyzed for the differences between the five treatments.

\begin{tabular}{lccc}
\hline \hline Dissimilarity & $F_{4,50}$ & $R^{2}$ & $P$ \\
\hline Taxonomic & 2.47 & 0.165 & 0.001 \\
Morphometric & 2.55 & 0.169 & 0.001 \\
Life history & 3.32 & 0.210 & 0.001 \\
\hline
\end{tabular}

Notes: Taxonomic dissimilarity are Bray-Curtis distances, morphometric and life-history dissimilarities are based on functional distances determined by the trait probability density framework. Results are based on 999 permutations.

3, which was strongly associated with Harvest only, consisted of xerophylic species that feed on mesophyll as feeding generalists. These species also tend to overwinter in later developmental stages (see Appendix S1: Table S9 for details). The results of RLQ analyses were consistent with the analyses of community weighted means (Appendix S1: Fig. S16).

\section{Discussion}

In this study, we investigated how effective different restoration measures were in restoring taxonomic and functional diversity and community of herbivorous insect $22 \mathrm{yr}$ after implementation. We expected to find a positive effect of all restoration treatments on all aspects of the insect communities considered, but hypothesized that more invasive restoration measures that include topsoil removal were more successful in restoring the target state. These hypotheses were supported by our results. Herbivorous insect communities sampled in Topsoil and Topsoil + Propagules were closer to the target state in terms of both diversity and composition compared to Harvest only, which still differed from Initial. In terms of functional composition, we expected that species with trait characteristics and trait combinations susceptible to land-use intensification could be restored, but again success would depend on the restoration measure implemented. Indeed, we identified distinct trait syndromes characterizing the different treatments, with species having trait combinations that respond particularly negatively to land-use intensification being more successfully restored by Topsoil and Topsoil + Propagules than by Harvest only.

\section{Success of restoration measures in re-establishing herbivore communities}

Taxonomic and functional diversity as well as composition approached the target state for all restoration measures, with more invasive measures involving topsoil removal being more successful in restoring the target state (Harvest only $<$ Topsoil, Topsoil + Propagules). These results are in line with previous studies conducted in agricultural landscapes showing that with extensification (i.e., mowing reduction) insect species diversity can be restored (Nickel and Achtziger 2005, Karg et al. 2015). While these studies focused on local taxonomic richness ( $\alpha$-diversity), we show that restoration measures are particularly valuable for restoring taxonomic diversity at the landscape level ( $\gamma$-diversity) and are equally successful for rare and dominant species (see diversity profiles; Fig. 1). However, for taxonomic $\alpha$-diversity, only Topsoil and Topsoil + Propagules had a positive effect, while Harvest only showed similarly low $\alpha$-diversity values as observed for Initial. This suggests that more invasive restoration measures are more effective in increasing diversity on the local level, while less invasive measures cause variability among plots and thus increase $\beta$-diversity. The response of functional diversity was similar to the one of taxonomic diversity, emphasizing that species and functional diversity were recovered 


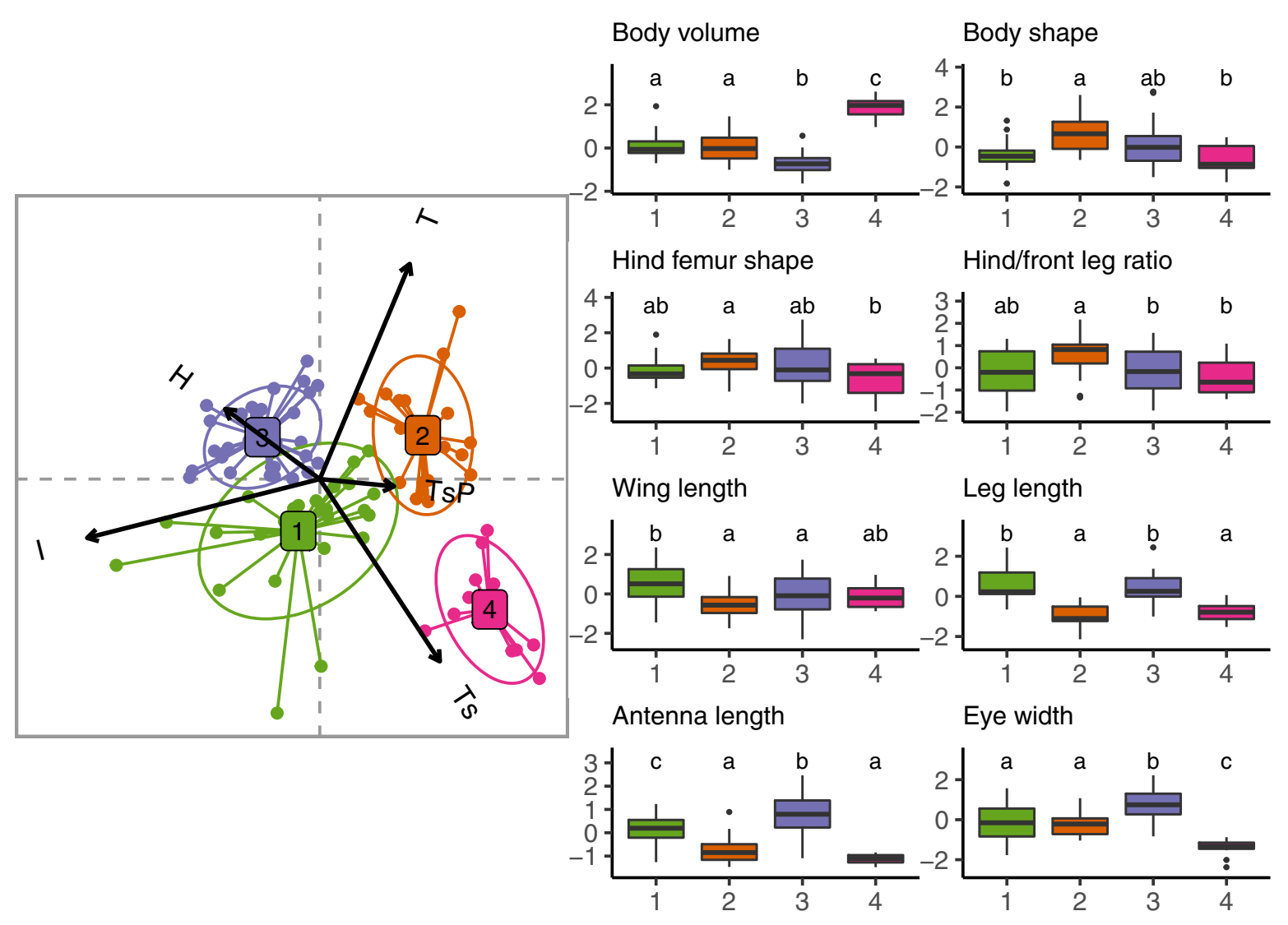

FIG. 3. RLQ analysis for morphometric traits. Left: Ordination biplot with each point representing a species. The colors refer to the four clusters, which were derived from hierarchical cluster analysis. The cluster ellipses were calculated based on a multivariate normal distribution with a confidence level of 0.66 . Arrows were rescaled for illustration purposes and show both strength and direction of treatment effects (I, Initial; H, Harvest only; Ts, Topsoil; TsP, Topsoil + Propagules; T, Target). Right: Scaled trait values for the four clusters for all eight morphometric traits. Significant differences (ANOVA, Tukey HSD) in trait values between clusters are indicated with letters. Box plot components are mid line (median), box edges (first and third quartiles), and whiskers (extend to the lowest and highest observed values that lie within 1.5 times the interquartile distance).

simultaneously. In other words, it indicates that environmental filters imposed by intensive land use are reducing the functional trait space occupied by the insect communities. Structural equation models showed that these environmental filters involved both direct effects of restoration measures on insect diversity as well as indirect effects mediated by the plant community. Direct effects might, for example, involve direct lethal incidents caused by regular mowing (Humbert et al. 2009), which is supported by our findings on trait syndromes with more mobile species being more strongly associated to Initial. Indirect effects through vegetation height and forb biomass might indicate the role of higher habitat and food plant diversity at Target as well as restoration sites (mainly Topsoil and Topsoil + Propagules) in supporting a higher taxonomic as well as functional diversity of herbivorous insects. This is supported by previous findings on the important role of the vegetation composition, with higher diversity in habitats being related to higher diversity of grassland arthropods (Stinson and Brown 1983, Gibson et al. 1992, Woodcock et al. 2007).
The large overlap in both taxonomic and functional composition between restoration measures and Target indicates that recovery of diversity also involves recovery of the taxonomic and functional identities of the target state. The patterns found for herbivore community composition strongly resemble those observed for plant communities in the same system (Resch et al. 2019). Moreover, our results suggest an important role of indirect pathways that link restoration treatments and herbivorous insect community composition through plant community composition. This indicates bottom-up control of the herbivores through the plant community, which has been reported from other restoration studies (Rotchés-Ribalta et al. 2018). As for diversity indices, the restoration measures that involved topsoil removal proved to be more effective in moving the community composition away from Initial and toward Target compared to Harvest only, which further supports our hypothesis that more invasive restoration measures are more effective in restoring the target state. The lower effects of Harvest only compared to the other two 

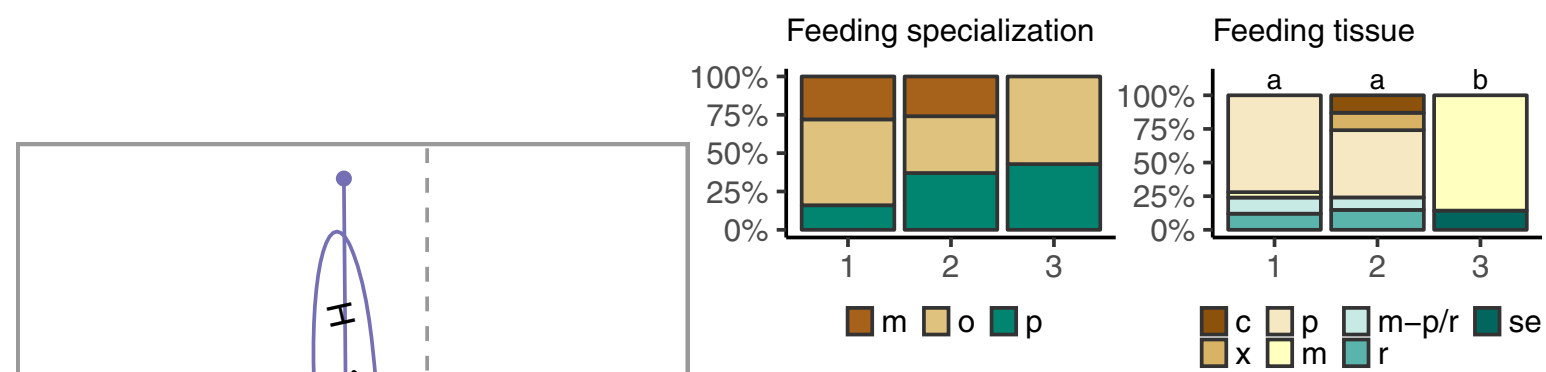

Hibernation stage

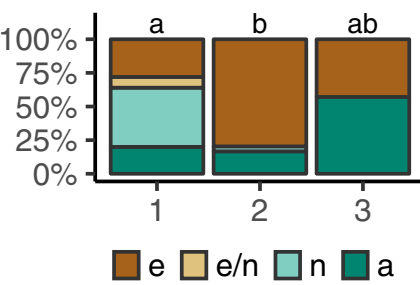

Moisture preference

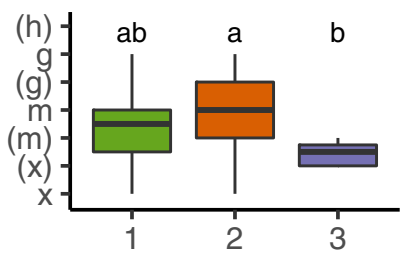

Generations

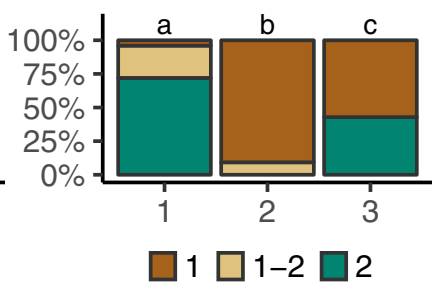

Moisture range

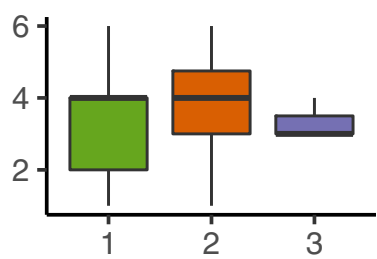

FIG. 4. RLQ analysis for life-history traits. Left: Ordination biplot with each point representing a species. The colors refer to the three clusters, which were derived from hierarchical cluster analysis. The cluster ellipses were calculated based on a multivariate normal distribution with a confidence level of 0.66. Arrows were rescaled for illustration purposes and show both strength and direction of treatment effects (I, Initial; H, Harvest only; Ts, Topsoil; TsP, Topsoil + Propagules; T, Target). Right: For discrete traits, the graphs show the distribution of trait values within each cluster. Feeding specialization (m, monophagous; o, oligophagous; $\mathrm{p}$, polyphagous), feeding tissue (c, chewers, $\mathrm{x}$, xylem, $\mathrm{p}$, phloem, m, mesophyll, m-p/r, mesophyll as well as phloem and/or reproductive organs; r, reproductive organs; se, ripe seeds), hibernation stage (e, egg; e/n, egg and nymph; n, nymph; a, adult), and number of generations per year are shown. For continuous traits (moisture preference, moisture range) the box plots show the scaled trait values for the three clusters. Box plot components are mid line (median), box edges (first and third quartiles), and whiskers (extend to the lowest and highest observed values that lie within 1.5 times the interquartile distance). The $y$-axis for moisture preference denotes the underlying moisture preference classification (x, xerophilous; m, mesohygrophilous; g, hygrophilous; brackets denote transition states). Moisture range indicates the specialization for a certain habitat moisture, with high values indicating moisture generalists. Significant differences in trait values between clusters are indicated with letters (ANOVA with Tukey-HSD for continuous trait values, pairwise chi-squared tests for discrete trait values).

restoration measures are probably due to land-use legacies (e.g., soil nutrient content). This supports previous findings that restoration measures in these systems need interventions such as topsoil removal to recover the target state (Verhagen et al. 2001). In contrast, differences between Topsoil and Topsoil + Propagules were found to be small, both for diversity and community composition; a finding that is in accordance with previous studies (Woodcock et al. 2010) and the results for plant and nematode communities at the same sites (Resch et al. 2019). Given that there was no clear difference in plant communities between the topsoil removal treatments and that treatment effects on insect composition were mainly mediated by the plant community, the close resemblance of Topsoil and Topsoil + Propagules insect communities is not surprising. However, there might still be advantages of propagule addition as it might accelerate restoration success especially within the first years after implementation (cf. Woodcock et al. 2012), but this issue was not addressed in this current project. In conclusion, this study is the first to show that positive effects of topsoil removal are not restricted to belowground taxa and plants (Frouz et al. 2009, Resch et al. 2019), but can encompass aboveground invertebrate communities as well.

\section{Effects of restoration measures in restoring trait} combinations being sensitive to land-use intensification

Trait combinations, which have previously been found to be particularly sensitive to environmental filters 
imposed by land-use intensification and were thus hypothesized to change accordingly, responded positive to restoration measures, in line with our third hypothesis. Herbivore species strongly associated to Initial sites were characterized by long wings and legs, indicating high dispersal and disturbance avoidance capabilities, which are advantageous in intensively managed ecosystems with regular disturbances, in particular mowing (Ribera et al. 2001, Börschig et al. 2013, Simons et al. 2016, Birkhofer et al. 2017). Disturbance avoidance might also explain that the Harvest only communities were characterized by small-bodied insects that overwinter in later developmental stages, which have previously been shown to be more prevalent under high land-use intensities (Ribera et al. 2001, Börschig et al. 2013, Rader et al. 2014, Simons et al. 2016, Birkhofer et al. 2017). Small body volume as well as overwintering in later developmental stages ensures individuals to reach the adult stage early in the year, which has the advantage of closing the life cycle before the first mowing. Also, they tend to have better developed dispersal abilities earlier in the year, which allows them to escape disturbances. In fact, the first mowing at Harvest only sites took place in late spring to early summer whereas Topsoil, Topsoil + Propagules, and Target sites were mowed in late summer or early autumn. At these latter sites, large-bodied herbivores with short legs and short antennae that produce one generation per year and overwinter in the egg stage were dominant. These are traits of species susceptible to regular and early-year disturbances, such as in Harvest only, because they could not complete their development. The short antennae indicate reduced tactile orientation ability, which might be needed less in open habitats that are potentially more prevalent in the restored sites.

Interestingly, we did not find species in the restoration measures and Target to be more specialized in their food choice than those associated with Initial, although previous studies showed lower amounts of feeding specialists with increasing land-use intensity (Rader et al. 2014, Simons et al. 2016). This phenomenon can be explained by a change of host specialists. While in Target and restoration sites many species specialized on particular forbs occurring, we found a high proportion of Auchenorrhyncha specialized on Poaceae being associated to Initial sites, which contained higher proportions of Poaceae (mean cover of $49.8 \%$ ) compared to the other treatments (18.4-33.5\%).

In sum, restoration measures, in particular those involving topsoil removal, were successful in promoting species with traits that are disadvantageous at high landuse intensities. This has, to our knowledge, rarely been shown for aboveground invertebrates (Verhagen et al. 2008). The fact that we did not find a difference in dispersal ability between Topsoil, Topsoil + Propagules and Target shows that $22 \mathrm{yr}$ after restoration, potential dispersal filters, which might hamper restoration (Öckinger et al. 2018), were successfully overcome.

\section{Conclusions}

This study shows that $22 \mathrm{yr}$ after restoration of intensively managed grasslands, insect herbivore communities were successfully restored, in particular by measures involving topsoil removal. At the same time, adding plant propagules to sites where topsoil was removed did not have much of an additional impact on herbivore communities. The close resemblance of community composition patterns found for plants and herbivore insects and the finding of strong indirect pathways on herbivores via changes in plant communities indicate bottom-up controls of the herbivore communities. This suggests that also for herbivore insects, removal of the topsoil layer and the nutrients contained therein is crucial for the long-term success of seminatural grassland restoration. For a management perspective, we recommend that restoration of seminatural grasslands on formerly intensively managed grassland should involve topsoil removal to be most effective in restoring multi-trophic communities in general and the functional and taxonomic composition of insect herbivore communities in particular. At which spatial scale (smaller patches as source populations and stepping stones vs. large-scale application) these measures should or can be applied for successful restoration of a particular landscape might depend on the landscape configuration as well as on ecological-economic trade-offs and needs to be evaluated by future studies.

\section{ACKNOWLEDGMENTS}

We thank Matthew Ayres and the anonymous reviewers for their valuable comments on earlier versions of the manuscript. We thank the Nature Conservation Agency of Canton Zurich, especially Pascale Weber and Ursina Wiedmer, for support in negotiating with farmers and regional commissioners for nature conservation, and access to the agency archives. We thank Matthias Diener for field assistance. We also thank Roland Mühlethaler and Tobias Meier for their species identification work. We are grateful to the Bavarian State Collection of Zoology, the Senckenberg Museum Dresden, A. Hilpert, and G. Kunz for providing specimens for morphometric measurements and to S. Berendt, L. Höck, M. Pfitzer, and E. Sackey for their help in taking those measurements. This study was funded by the Swiss National Science Foundation (SNF) grant-no 31003A_166654 to M. Schütz and A. C. Risch. F. Neff and M. M. Gossner obtained funding from SNF grant-no 310030E173542/1. The authors contributed as follows to this study: F. Neff, M. C. Resch, M. Schütz, A. C. Risch, and M. M. Gossner conceived and developed the ideas for the manuscript; M. C. Resch, M. Schütz, and A. C. Risch designed the experiment; M. C. Resch, A. Marty, J. D. Rolley, and M. M. Gossner collected the data; F. Neff and M. M. Gossner analyzed the data; F. Neff, M. C. Resch, and M. M. Gossner wrote the first draft; all authors contributed critically to the drafts and gave final approval for publication.

\section{Literature Cited}

Allison, M., and M. Ausden. 2004. Successful use of topsoil removal and soil amelioration to create heathland vegetation. Biological Conservation 120:221-228. 
Belyea, L. R., and J. Lancaster. 1999. Assembly rules within a contingent ecology. Oikos 86:402-416.

Birkhofer, K., et al. 2017. Land-use type and intensity differentially filter traits in above- and below-ground arthropod communities. Journal of Animal Ecology 86:511-520.

Börschig, C., A.-M. Klein, H. von Wehrden, and J. Krauss. 2013. Traits of butterfly communities change from specialist to generalist characteristics with increasing land-use intensity. Basic and Applied Ecology 14:547-554.

Caliński, T., and J. Harabasz. 1974. A dendrite method for cluster analysis. Communications in Statistics 3:1-27.

Carmona, C. P. 2017. Methods for measuring functional diversity based on trait probability density. $\mathrm{R}$ package version 0.1.2. https://CRAN.R-project.org/package $=$ TPD

Carmona, C. P., F. de Bello, N. W. H. Mason, and J. Lepš. 2016. Traits without borders: Integrating functional diversity across scales. Trends in Ecology and Evolution 31:382-394.

Chao, A., C.-H. Chiu, and L. Jost. 2014. Unifying species diversity, phylogenetic diversity, functional diversity, and related similarity and differentiation measures through Hill Numbers. Annual Review of Ecology, Evolution, and Systematics 45:297-324.

Chao, A., C.-H. Chiu, S. Villéger, I.-F. Sun, S. Thorn, Y.-C. Lin, J.-M. Chiang, and W. B. Sherwin. 2019. An attribute-diversity approach to functional diversity, functional beta diversity, and related (dis)similarity measures. Ecological Monographs 89:e01343.

Chao, A., and L. Jost. 2012. Coverage-based rarefaction and extrapolation: standardizing samples by completeness rather than size. Ecology 93:2533-2547.

Delarze, R., Y. Gonseth, S. Eggenberg, and M. Vust. 2015. Lebensräume der Schweiz: Ökologie - Gefährdung - Kennarten. Third edition. Ott, Bern, Switzerland

Dolédec, S., D. Chessel, C. J. F. ter Braak, and S. Champely. 1996. Matching species traits to environmental variables: a new three-table ordination method. Environmental and Ecological Statistics 3:143-166.

Dray, S., A.-B. Dufour, and J. Thioulouse. 2018. Analysis of ecological data: exploratory and Euclidean methods in environmental sciences. $\mathrm{R}$ package version 1.7-11. https:// CRAN.R-project.org/package $=$ ade4

Frouz, J., R. Van Diggelen, V. Pižl, J. Starý, L. Háněl, K. Tajovský, and J. Kalčík. 2009. The effect of topsoil removal in restored heathland on soil fauna, topsoil microstructure, and cellulose decomposition: implications for ecosystem restoration. Biodiversity and Conservation 18:3963-3978.

Gámez-Virués, S., et al. 2015. Landscape simplification filters species traits and drives biotic homogenization. Nature Communications 6:8568.

Gattlen, N., G. Klaus, and G. Litsios. 2017. Biodiversität in der Schweiz: Zustand und Entwicklung. Ergebnisse des Überwachungssystems im Bereich Biodiversität, Stand 2016. Bundesamt für Umwelt, Bern, Switzerland.

Gibson, C. W. D., V. K. Brown, L. Losito, and G. C. McGavin. 1992. The response of invertebrate assemblies to grazing. Ecography 15:166-176.

Gimmi, U., T. Lachat, and M. Bürgi. 2011. Reconstructing the collapse of wetland networks in the Swiss lowlands 1850 2000. Landscape Ecology 26:1071.

Gossner, M. M., et al. 2016. Land-use intensification causes multitrophic homogenization of grassland communities. Nature 540:266-269.

Gossner, M. M., N. K. Simons, R. Achtziger, T. Blick, W. H. O. Dorow, F. Dziock, F. Köhler, W. Rabitsch, and W. W. Weisser. 2015. A summary of eight traits of Coleoptera, Hemiptera, Orthoptera and Araneae, occurring in grasslands in Germany. Scientific Data 2:150013.
Habel, J. C., A. Segerer, W. Ulrich, O. Torchyk, W. W. Weisser, and T. Schmitt. 2016. Butterfly community shifts over two centuries. Conservation Biology 30:754-762.

Hallmann, C. A., et al. 2017. More than 75 percent decline over 27 years in total flying insect biomass in protected areas. PLoS ONE 12:e185809.

Humbert, J.-Y., J. Ghazoul, and T. Walter. 2009. Meadow harvesting techniques and their impacts on field fauna. Agriculture, Ecosystems \& Environment 130:1-8.

IPBES. 2019. Summary for policymakers of the global assessment report on biodiversity and ecosystem services of the Intergovernmental Science-Policy Platform on Biodiversity and Ecosystem Services. IPBES secretariat, Bonn, Germany.

Karg, J., K. Kujawa, C. Manhart, H. Marschalek, K. R. Neugebauer, and J. Sachteleben. 2015. Restoration of subalpine species-rich grasslands: short-term vs long-term changes in the fensity and fiversity of above-ground insects. Polish Journal of Ecology 63:142-158.

Keddy, P. A. 1992. Assembly and response rules: two goals for predictive community ecology. Journal of Vegetation Science 3:157-164.

Kiehl, K., A. Kirmer, T. W. Donath, L. Rasran, and N. Hölzel. 2010. Species introduction in restoration projects-Evaluation of different techniques for the establishment of semi-natural grasslands in Central and Northwestern Europe. Basic and Applied Ecology 11:285-299.

Kiehl, K., and J. Pfadenhauer. 2007. Establishment and persistence of target species in newly created calcareous grasslands on former arable fields. Plant Ecology 189:31-48.

Kleyer, M., S. Dray, F. de Bello, J. Lepš, R. J. Pakeman, B. Strauss, W. Thuiller, and S. Lavorel. 2012. Assessing species and community functional responses to environmental gradients: Which multivariate methods? Journal of Vegetation Science 23:805-821.

Laliberté, E., P. Legendre, and B. Shipley. 2014. Measuring functional diversity (FD) from multiple traits, and other tools for functional ecology. R package version 1.0-12. https:// CRAN.R-project.org/package $=F D$

Lamanna, C., et al. 2014. Functional trait space and the latitudinal diversity gradient. Proceedings of the National Academy of Sciences USA 111:13745-13750.

Lefcheck, J. S. 2016. piecewiseSEM: Piecewise structural equation modelling in $\mathrm{r}$ for ecology, evolution, and systematics. Methods in Ecology and Evolution 7:573-579.

Manning, P., et al. 2015. Grassland management intensification weakens the associations among the diversities of multiple plant and animal taxa. Ecology 96:1492-1501.

Marrs, R. H., C. S. R. Snow, K. M. Owen, and C. E. Evans. 1998. Heathland and acid grassland creation on arable soils at Minsmere: identification of potential problems and a test of cropping to impoverish soils. Biological Conservation 85:69-82.

McGill, B. J., B. J. Enquist, E. Weiher, and M. Westoby. 2006. Rebuilding community ecology from functional traits. Trends in Ecology and Evolution 21:178-185.

MeteoSchweiz. 2018. Klimabulletin Jahr 2017. MeteoSchweiz, Zürich, Switzerland.

Neff, F., N. Blüthgen, M. N. Chisté, N. K. Simons, J. Steckel, W. W. Weisser, C. Westphal, L. Pellissier, and M. M. Gossner. 2019. Cross-scale effects of land use on the functional composition of herbivorous insect communities. Landscape Ecology 34:2001-2015.

Newbold, T., et al. 2015. Global effects of land use on local terrestrial biodiversity. Nature 520:45-50.

Nickel, H., and R. Achtziger. 2005. Do they ever come back? Responses of leafhopper communities to extensification of land use. Journal of Insect Conservation 9:319-333. 
Öckinger, E., A. K. Eriksson, and H. G. Smith. 2006. Effects of grassland abandonment, restoration and management on butterflies and vascular plants. Biological Conservation 133:291-300.

Öckinger, E., M. Winsa, S. P. M. Roberts, and R. Bommarco. 2018. Mobility and resource use influence the occurrence of pollinating insects in restored seminatural grassland fragments. Restoration Ecology 26:873-881.

Oksanen, J., et al. 2018. Community ecology package. R package version 2.5-2. https://CRAN.R-project.org/package=vegan

Paradis, E., et al. 2019. Analyses of phylogenetics and evolution. R package version 5.3. https://CRAN.R-project.org/pac kage $=$ ape

Poschlod, P., and M. F. WallisDeVries. 2002. The historical and socioeconomic perspective of calcareous grasslands-lessons from the distant and recent past. Biological Conservation 104:361-376.

Power, S. A., E. R. Green, C. G. Barker, J. N. B. Bell, and M. R. Ashmore. 2006. Ecosystem recovery: heathland response to a reduction in nitrogen deposition. Global Change Biology 12:1241-1252.

R Core Team. 2018. R: a language and environment for statistical computing. R Foundation for Statistical Computing, Vienna, Austria.

Rader, R., I. Bartomeus, J. M. Tylianakis, E. Laliberté, and M. van Kleunen. 2014. The winners and losers of land use intensification: pollinator community disassembly is non-random and alters functional diversity. Diversity and Distributions 20:908-917.

Resch, M. C., M. Schütz, U. Graf, R. Wagenaar, W. H. van der Putten, and A. C. Risch. 2019. Does topsoil removal in grassland restoration benefit both soil nematode and plant communities? Journal of Applied Ecology 56:1782-1793.

Ribera, I., S. Dolédec, I. S. Downie, and G. N. Foster. 2001. Effect of land disturbance and stress on species traits of ground beetle assemblages. Ecology 82:1112-1129.

Rotchés-Ribalta, R., M. Winsa, S. P. M. Roberts, and E. Öckinger. 2018. Associations between plant and pollinator communities under grassland restoration respond mainly to landscape connectivity. Journal of Applied Ecology 55:28222833.

Rowe, H. I., and J. D. Holland. 2013. High plant richness in prairie reconstructions support diverse leafhopper communities. Restoration Ecology 21:174-180.

Sala, O. E., et al. 2000. Global biodiversity scenarios for the year 2100. Science 287:1770-1774.

Sánchez-Bayo, F., and K. A. G. Wyckhuys. 2019. Worldwide decline of the entomofauna: A review of its drivers. Biological Conservation 232:8-27.
Seibold, S., et al. 2019. Arthropod decline in grasslands and forests is associated with landscape-level drivers. Nature 574:671-674

Simons, N. K., W. W. Weisser, and M. M. Gossner. 2016. Multitaxa approach shows consistent shifts in arthropod functional traits along grassland land-use intensity gradient. Ecology 97:754-764.

Soliveres, S., et al. 2016. Biodiversity at multiple trophic levels is needed for ecosystem multifunctionality. Nature 536:456459

Stinson, C. S. A., and V. K. Brown. 1983. Seasonal changes in the architecture of natural plant communities and its relevance to insect herbivores. Oecologia 56:67-69.

Temme, A. J. A. M., and P. H. Verburg. 2011. Mapping and modelling of changes in agricultural intensity in Europe. Agriculture, Ecosystems \& Environment 140:46-56.

Török, P., and J. Dengler 2018. Palaearctic grasslands in transition: overarching patterns and future prospects. Pages 15-26 in V. R. Squires, J. Dengler, H. Feng, and L. Hua, editors. Grasslands of the world: diversity, management and conservation. CRC Press, Boca Raton, Florida, USA.

Verhagen, R., J. Klooker, J. P. Bakker, and R. van Diggelen. 2001. Restoration success of low-production plant communities on former agricultural soils after top-soil removal. Applied Vegetation Science 4:75-82.

Verhagen, R., R. van Diggelen, and R. Vermeulen. 2008. Community assemblage of the Carabidae fauna in newly created habitats. Baltic Journal of Coleopterology 8:135-148.

WallisDeVries, M. F., and I. Raemakers. 2001. Does extensive grazing benefit butterflies in coastal dunes? Restoration Ecology 9:179-188.

Woodcock, B. A., et al. 2010. The role of management and landscape context in the restoration of grassland phytophagous beetles. Journal of Applied Ecology 47:366-376.

Woodcock, B. A., J. M. Bullock, S. R. Mortimer, and R. F. Pywell. 2012. Limiting factors in the restoration of UK grassland beetle assemblages. Biological Conservation 146:136-143.

Woodcock, B. A., S. G. Potts, D. B. Westbury, A. J. Ramsay, M. Lambert, S. J. Harris, and V. K. Brown. 2007. The importance of sward architectural complexity in structuring predatory and phytophagous invertebrate assemblages. Ecological Entomology 32:302-311.

Young, T. P., D. A. Petersen, and J. J. Clary. 2005. The ecology of restoration: historical links, emerging issues and unexplored realms. Ecology Letters 8:662-673.

Zirbel, C. R., E. Grman, T. Bassett, and L. A. Brudvig. 2019. Landscape context explains ecosystem multifunctionality in restored grasslands better than plant diversity. Ecology 100: $\mathrm{e} 02634$.

\section{SUPPORTING INFORMATION}

Additional supporting information may be found online at: http://onlinelibrary.wiley.com/doi/10.1002/eap.2133/full

Data Availability

Data are available from the EnviDat Repository: https://doi.org/10.16904/envidat.142 